\title{
Relaxation of polydisperse electrorheological suspensions
}

\author{
K. H. Ahn and D. J. Klingenberg \\ University of Wisconsin, Department of Chemical Engineering and \\ Rheology Research Center, 1415 Johnson Drive, Madison, Wisconsin 53706
}

(Received 20 August 1993; accepted 11 February 1994)

\begin{abstract}
Synopsis
Relaxation of monodisperse and polydisperse electrorheological (ER) suspensions under linear deformation is investigated. The electrostatic polarization model is employed to describe ER suspensions, and solutions to the equations of motion are obtained by dynamic simulation and regular perturbation methods. Sources of relaxation events are identified by examining partial rheological functions that are defined in the text. Relaxation of both monodisperse and polydisperse suspensions is found to be confined to approximately the same limited range of relaxation times, although polydisperse suspensions may produce relaxation events over a very broad range of relaxation times. Events occurring at large relaxation times arise from relatively weak forces that do not significantly influence the extent of relaxation. Other relaxation mechanisms and potential sources of dispersion broadening are also discussed.
\end{abstract}

\section{INTRODUCTION}

The electrostatic polarization model of the electrorheological (ER) response envisions ER suspensions to be composed of class A dielectric particles immersed in a class A dielectric continuous phase. The applied electric field induces anisotropic, essentially dipolar, electrostatic interactions between particles, suggesting that the particles should aggregate into columns that span the electrode gap, as commonly observed in many ER systems. The form of the Maxwell's stress tensor also suggests that should a yield stress arise from the electrostatic forces, this yield stress will vary as the square of the electric field strength, again in agreement with many experimental observations.

This model for ER suspensions has been employed in several particle-level, molecular dynamics-like simulation studies of the steady shear response [Bailey et al. (1989); Klingenberg et al. (1989); Bonnecaze and Brady (1990); Whittle (1990); Klingenberg et al. (1991a,b); Bonnecaze and Brady (1992a,b); Melrose (1992); See and Doi (1992)]. Such studies confirm the expected field-induced formation of particulate chains, as well as the squared field strength dependence of the dynamic yield stress. Perhaps more significantly, however, is that these simulations offer insight into the microscopic mechanisms controlling ER behavior. For example, simulations at low shear rates show that shear stress transfer is controlled by the continuous breaking and reforming of the particulate strands, which had been speculated many times previously. The magnitude of the dynamic yield stress was shown, in simulation studies, to be sensitive to the interparticle forces at short range [Klingenberg et al. (1991b); Bonnecaze and Brady (1992b)]. Simulations first predicted that the dynamic yield stress should saturate above a critical particle concentration, which was later confirmed by experiments [Klingenberg et al. (1991a,b)]. The mecha- 
nism for saturation was identified in simulation studies, where it was shown that thicker clusters formed at large concentrations are less effective at transmitting electrostatic stress from one electrode to the other. Identifying mechanisms that control the steady shear response is valuable for improving ER suspensions and devices, for instance, by determining the physical properties and geometries that provide the optimum response for specific applications.

Devices employing ER technology, including engine mounts, clutches, brakes, and shock absorbers, typically operate in transient or periodic modes, as opposed to steady shear. Compared to the steady-shear response, little progress has been made in understanding transient responses in ER, and the underlying microscopic mechanisms. In fact, experimental studies of the dynamic response have produced varied and sometimes conflicting results [see Klingenberg (1993) and references therein for examples of conflicting experimental results]. Developing ER technology to its full potential is inhibited by the current deficit in the understanding of dynamic properties.

In a previous paper [Klingenberg (1993), referred to hereafter as Paper I], the dynamic simulation technique was employed to investigate the dynamic oscillatory response of ER suspensions. The main conclusions from this work, relevant to the linear response of monodisperse ER suspensions conforming to the electrostatic polarization model, were: (1) the dynamic response possesses the property of time-field strength superposition, (2) relaxation arises from the competition between electrostatic and hydrodynamic forces that produces a frequency-dependent dynamic structure, and (3) this relaxation occurs over a fairly narrow frequency range. The property of time-field strength superposition has been confirmed experimentally [Parthasarathy et al. (1993)]. However, most experimental data reported in the literature do not show a sharp dispersion. In Paper I, several possible mechanisms for dispersion broadening were suggested. These included particle size and shape polydispersity, and nonlinearity. Polydispersity was expected to contribute to dispersion broadening because the magnitude of the electrostatic interaction between two spheres depends sensitively on the relative sizes of the two spheres. Otsubo et al. (1992) experimentally observed a pronounced broadening of the dispersion as the oscillatory strain amplitude was increased from $1 \%$ to $20 \%$, suggesting that nonlinearity may provide another mechanism for dispersion broadening. This has been supported by recent experiments and simulations that compared the frequency dependence of the shear moduli at various strain amplitudes [Parthasarathy et al. (1993)].

In this paper, we investigate the role of particle size polydispersity on the dynamic response of ER suspensions, in particular, to determine the influence of polydispersity on the breadth of dispersion. In Sec. II, we briefly review the dynamic simulation method, and introduce several new techniques for probing the behavior of idealized suspensions. Simulated shear stress relaxation is found to be computationally more efficient than simulation of oscillatory shear flow, and provides precisely the same rheological information for small amplitude deformations. The idealized microscopic model for ER suspensions that has previously been solved by dynamic simulation techniques is also amenable to regular perturbation solution methods; one such method is introduced in Sec. II, then developed and solved in detail for a specific suspension structure in the Appendix. This technique is also computationally efficient, and is valid for arbitrary, small amplitude deformations. Partial rheological functions are also introduced in Sec. II. These functions arise naturally from the form of the direct electrostatic stress, and are valuable for determining the microscopic features that produce various relaxation events. The results of this investigation are presented in Sec. III. The dynamic response of monodisperse ER suspensions is revisited in detail, in order to demonstrate that the relaxation mechanism identified in Paper I depends in a complicated manner on the positions of all 
of the spheres, and that the characteristic relaxation times can vary over a limited frequency range. The influence of polydispersity is investigated in Sec. III B. Here it is shown that while the presence of different size spheres can produce relaxation events over a very broad range of frequencies, the impact of these events on the extent of relaxation is confined to a relatively narrow range. As a result, polydispersity is not expected to significantly alter the dispersion exhibited by monodisperse suspensions under small amplitude deformation, and hence other mechanisms must be responsible for the broad dispersions observed experimentally. Candidate mechanisms are briefly discussed in Sec. IV, along with a summary of the major conclusions of this investigation.

\section{THEORY}

\section{A. Governing equations}

In this paper, we employ the idealized electrostatic polarization model for ER suspensions that has been implemented previously in simulations of monodisperse systems [Klingenberg (1992a); Paper I]. Here we extend this model to account for polydisperse suspensions in transient flows. ER suspensions are treated as neutrally buoyant, hard, class A dielectric spheres (dielectric constant $\epsilon_{p}$ ) immersed in a class A dielectric, Newtonian continuous phase (dielectric constant $\epsilon_{c}$, viscosity $\eta_{c}$ ). All the spheres possess the same dielectric constant, but can have different diameters. Application of an electric field polarizes the spheres, inducing electrostatic interactions between them, and between each sphere and the electrodes. The continuous phase is treated as a continuum; its presence influences only the magnitude of the electrostatic interactions and the hydrodynamic resistance on each sphere. For large particles under large electric fields, colloidal and Brownian forces are negligible [Marshall et al. (1989)].

Ignoring inertia terms, the motion of sphere $i$ not near an electrode is governed by

$$
\mathbf{F}_{i}\left(\left\{\mathbf{R}_{j}\right\}\right)=0
$$

where $\mathbf{F}_{i}\left(\left\{\mathbf{R}_{j}\right\}\right)$ is the resultant force acting on sphere $i$ due to the contributions described above, which depend on the positions of all of the spheres. Equation (1) represents a coupled set of equations governing the motion of all of the spheres not near electrodes (the motion of spheres near electrodes is treated below). Solving these equations requires explicit expressions for the forces.

The electrostatic force on sphere $i$ (diameter $\sigma_{i}$ ) at the origin of a spherical coordinate system due to sphere $j$ (diameter $\left.\sigma_{j}\right)$ at $\left(R_{i j}, \theta_{i j}\right)$ (see Fig. 1) is treated in the point-dipole limit (Paper I):

$$
\mathbf{F}_{i j}^{\mathrm{el}}\left(R_{i j}, \theta_{i j}\right)=F_{s}\left(\sigma_{i}, \sigma_{j}\right)\left(\frac{R_{\min }}{R_{i j}}\right)^{4}\left[\left(3 \cos ^{2} \theta_{i j}-1\right) \mathbf{e}_{r}+\sin 2 \theta_{i j} \mathbf{e}_{\theta}\right],
$$

where $R_{\min }=\left(\sigma_{i}+\sigma_{j}\right) / 2$,

$$
F_{s}\left(\sigma_{i}, \sigma_{j}\right)=\frac{3}{16} \pi \epsilon_{0} \epsilon_{c} \beta^{2} E_{0}^{2}\left(\sigma_{j}^{2} \frac{16 \lambda_{i j}^{3}}{\left(1+\lambda_{i j}\right)^{4}}\right),
$$

$\epsilon_{0}=8.8542 \times 10^{-12} \mathrm{~F} / \mathrm{m}, \beta=\left(\epsilon_{p}-\epsilon_{c}\right) /\left(\epsilon_{p}+2 \epsilon_{c}\right)$, and $\lambda_{i j}=\sigma_{i} / \sigma_{j}$.

The hydrodynamic force on sphere $i$ is represented by Stokes' drag,

$$
\mathbf{F}_{i}^{\text {hyd }}\left(\mathbf{R}_{i}\right)=-3 \pi \eta_{c} \sigma_{i}\left(\frac{d \mathbf{R}_{i}}{d t}-\mathbf{u}^{\infty}\left(\mathbf{R}_{i}\right)\right)
$$

where $\mathbf{u}^{\infty}\left(\mathbf{R}_{i}\right)$ is the ambient fluid velocity. 

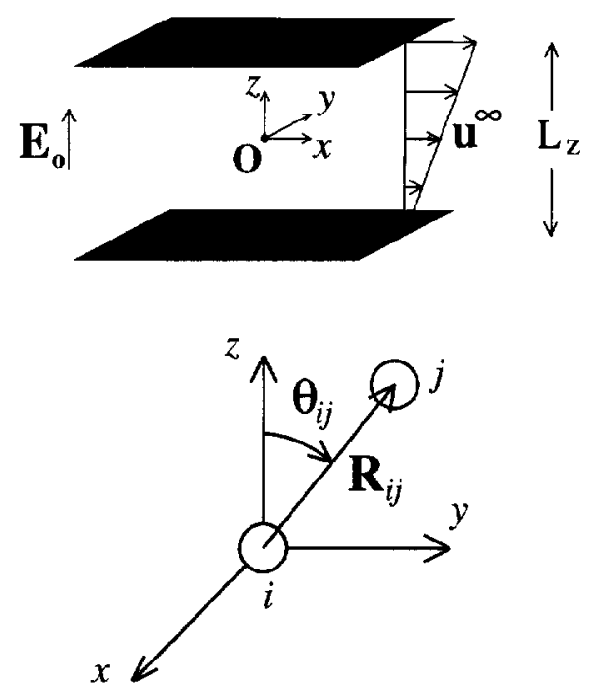

FIG. 1. Schematic diagram showing the electrode and sphere-pair geometries. The bottom electrode is held fixed and the top electrode is displaced in the $\pm x$ direction. $\mathbf{R}_{i j}$ is the center-to-center separation vector, and $\theta_{i j}$ is the angle between the line of centers and the applied electric field.

The hard-sphere interaction between spheres $i$ and $j$ is represented by a short-range repulsive force,

$$
\mathbf{F}_{i j}^{\mathrm{rep}}\left(\mathbf{R}_{i j}\right)=-F_{s}\left(\sigma_{i}, \sigma_{j}\right) \exp \left(-\kappa \frac{R_{i j}-R_{\min }}{R_{\min }}\right) \mathbf{e}_{r},
$$

where $\kappa$ characterizes the range of the repulsive interaction. The hard-sphere/hard-wall interaction is represented by a similar short-range function,

$$
\mathbf{F}_{i, \text { wall }}^{\mathrm{rep}}\left(\mathbf{R}_{i}\right)=\frac{3}{16} \pi \epsilon_{0} \epsilon_{c} \sigma_{i}^{2} \beta^{2} E_{0}^{2} \exp \left(-\kappa \frac{H_{i}-\sigma_{i} / 2}{\sigma_{i}}\right) \mathbf{n},
$$

where $H_{i}=L_{z} / 2-\left|z_{i}\right|, L_{z}$ is the electrode gap width, and $\mathbf{n}$ is the unit normal vector directed from the electrode into the suspension. Unless specifically stated otherwise, $\kappa$ is kept constant at $10^{2}$.

Incorporating these forces into Eq. (1), the equation of motion for sphere $i$ is written

$$
\begin{aligned}
3 \pi \eta_{c} \sigma_{i} \frac{d \mathbf{R}_{i}}{d t}= & \sum_{j \neq i} \mathbf{F}_{i j}^{\mathrm{el}}\left(R_{i j}, \theta_{i j}\right)+\sum_{j} \mathbf{F}_{i j}^{\mathrm{el}}\left(R_{i j}^{\prime}, \theta_{i j}^{\prime}\right)+\sum_{j \neq i}^{\prime \prime} \mathbf{F}_{i j}^{\mathrm{rep}}\left(R_{i j}, \theta_{i j}\right) \\
& +3 \pi \eta_{c} \sigma_{i} \mathbf{u}^{\infty}\left(\mathbf{R}_{i}\right) .
\end{aligned}
$$

The primes in the second summation indicate the electrostatic force between sphere $i$ and the image of sphere $j$; these forces are summed over all images of all spheres, including those of sphere $i$. The double prime in the third summation indicates that repulsive forces are evaluated between sphere $i$ and all spheres $j \neq i$, as well as between sphere $i$ and the electrodes. Equation (7) governs the motion of spheres not near an electrode surface. Spheres within $\delta_{w}=0.05 \sigma_{i}$ of an electrode are considered stuck, and assume the lateral 
velocity of the electrode. This sticking condition is based on experimental observation [Klingenberg and Zukoski (1990)]; as discussed previously within the framework of the present model, sticking at the electrodes is required to observe a yield stress [Klingenberg et al. (1991a)] and a storage modulus in the limit of zero frequency (Paper I). Implications regarding the possibility of slip at the electrodes are discussed in Sec. IV. The equations governing the motion of stuck spheres in polydisperse suspensions are given below in dimensionless form.

The equation of motion is made dimensionless with the following length, force, and time scales:

$$
l_{\text {scale }}=\sigma_{0}, \quad F_{\text {scale }}=\frac{3}{16} \pi \epsilon_{0} \epsilon_{c} \sigma_{0}^{2} \beta^{2} E_{0}^{2}, \quad t_{\text {scale }}=\frac{16 \eta_{c}}{\epsilon_{0} \epsilon_{c} \beta^{2} E_{0}^{2}},
$$

where $\sigma_{0}$ is the diameter of a reference sphere, taken to be that of the largest sphere. The equation of motion in dimensionless form for sphere $i$ not near an electrode is written

$$
\frac{d \mathbf{r}_{i}^{*}}{d t^{*}}=\frac{1}{\sigma_{i}^{*}}\left(\sum_{j \neq i} \mathbf{f}_{i j}^{\mathrm{e} * *}+\sum_{j} \mathbf{f}_{i j}^{\mathrm{el} *}+\sum_{j \neq i}^{\prime \prime} \mathbf{f}_{i j}^{\mathrm{rep} *}\right)+\mathbf{u}^{\infty} *\left(\mathbf{r}_{i}^{*}\right),
$$

where the asterisks denote dimensionless quantities.

The equations governing the motion of stuck spheres are written

$$
\begin{gathered}
\frac{d x_{i}^{*}}{d t^{*}}=u_{x, \text { elec }}^{*}, \\
\frac{d y_{i}^{*}}{d t^{*}}=u_{y, \text { elec }}^{*}, \\
\frac{d z_{i}^{*}}{d t^{*}}=\frac{1}{\sigma_{i}^{*}}\left(\sum_{j \neq i} f_{z, i j}^{\mathrm{el} *}+\sum_{j} f_{z, i j}^{\mathrm{el} * \prime}+\sum_{j \neq i}^{\prime \prime} f_{z, i j}^{\mathrm{rep} *}\right)+u_{z, \text { elec }}^{*},
\end{gathered}
$$

where $u_{k \text {,elec }}$ is the $k$ th component of the electrode velocity. Motion normal to the electrode surface is not directly influenced by this sticking condition. Undet large deformations, spheres are occasionally pulled away from the electrodes by neighboring spheres; when the distance between the electrode and the sphere surfaces exceeds $\delta_{w}^{*}$, the sphere can translate parallel to the electrode. Although this constitutes a possible mechanism for relaxation, it has not been observed for small deformations (strain amplitude $\leqslant 10^{-4}$ ). This issue is addressed again in Sec. IV.

In this paper, we consider three different ambient flow profiles: No flow:

$$
\mathbf{u}^{\infty *}\left(\mathbf{r}_{i}^{*}\right)=\mathbf{0}
$$

Oscillatory shear flow:

$$
\mathbf{u}^{\infty *}\left(\mathbf{r}_{i}^{*}\right)=\omega^{*} \gamma_{0}\left(z_{i}^{*}+\frac{L_{z}^{*}}{2}\right) \cos \omega^{*} t^{*} e_{x}
$$

Shear stress relaxation:

$$
\mathbf{u}^{\infty} *\left(\mathbf{r}_{i}^{*}\right)=\mathbf{0}, \quad t^{*}<0
$$




$$
\mathbf{u}^{\infty} *\left(\mathbf{r}_{i}^{*}\right)=\dot{\gamma}^{*}\left(z_{i}^{*}+\frac{L_{z}^{*}}{2}\right) \mathbf{e}_{x}, \quad 0<t^{*}<t_{0}^{*},
$$

and

$$
\mathbf{u}^{\infty *}\left(\mathbf{r}_{i}^{*}\right)=0, \quad t^{*}>t_{0}^{*}
$$

$\omega^{*}$ is the dimensionless frequency and $\gamma_{0}$ is the strain amplitude for oscillatory shear flow, $\dot{\gamma}^{*}$ is the dimensionless shear rate for stress relaxation, and $\dot{\gamma}^{*} t_{0}^{*}=\gamma_{0}^{\text {step }}$ is the step shear strain amplitude. To represent an instantaneous step strain, a large value of $\dot{\gamma}^{*}$ must be employed; we find that $\dot{\gamma}^{*} \geqslant 10^{3}$ is adequate to represent an instantaneous step strain deformation. The validity of this approximation is verified below, where rheological functions obtained from both small amplitude oscillation and stress relaxation are compared.

For convenience, the asterisks denoting dimensionless quantities will be dropped from here forward. All quantities will be dimensionless unless specifically stated otherwise.

\section{B. Solving the equations of motion}

Equations (8)-(11) represent a microscopic model for ER suspensions. Dimensional analysis of these equations provides scaling relationships among various quantities [e.g., time-field strength superposition (Paper I)], but further investigation of suspension behavior requires solutions to these equations to obtain the sphere positions as a function of time, and subsequently the rheological properties. In this paper, we employ two methods of solution: dynamic simulation and regular perturbation. These methods are described below.

\section{Simulation method}

The dynamic simulation technique employs direct numerical integration of Eqs. (8)(11). $N$ spheres are placed between electrodes at $z= \pm L_{2} / 2$, and within periodic boundaries at $x= \pm L_{x} / 2$, and $y= \pm L_{y} / 2$. Simulations in two dimensions are performed by placing all spheres in a plane $y=$ constant. Spheres are placed randomly or in a prescribed, manufactured configuration. Equations (8)-(11) are integrated numerically for each sphere using Euler's method, with a time step $\Delta t \leqslant \sigma_{\min } 10^{-3}$. Forces are evaluated within a cutoff radius $r_{c}=L_{z} / 2$. For rheological simulations, a metastable structure is first obtained by integrating the equations of motion under no flow until motion ceases. After the metastable structure is obtained, simulation under the desired flow is begun. For oscillatory shear, the simulations are performed until all transients decay, and the motion of each sphere is periodic. For stress relaxation simulations, a large shear rate is applied to give the desired strain, the shear rate is then set to zero, and the structure is allowed to relax under no flow until motion ceases. For both oscillatory shear and stress relaxation, responses were linear for strain amplitudes $\leqslant 10^{-4}$.

\section{Regular perturbation methods}

The dynamic simulation technique described above tends to require a large amount of computer time, especially when simulating the oscillatory response of suspensions containing particles very different in size; the presence of the small spheres requires small time steps, and many steps are required before transients decay. However, small amplitude deformations, such as those investigated here, are amenable to treatment by perturbation methods. These methods result in a set of linear ordinary differential equations that 
can be solved directly to give the time-dependent particle positions. The general method is developed in the Appendix, along with the detailed solution for a particular configuration of spheres.

\section{Evaluating rheological properties}

Once the sphere positions have been determined as a function of time, the rheological properties can be evaluated. The total stress is conveniently expressed as a sum of direct hydrodynamic and electrostatic contributions (Paper I),

$$
\boldsymbol{\sigma}^{\text {total }}=\boldsymbol{\sigma}^{E}+\boldsymbol{\sigma}^{H}
$$

This distinction between the contributions is arbitrary, but is chosen because the hydrodynamic contribution vanishes as the deformation rate vanishes or when the solvent is removed, whereas the electrostatic contribution may remain in both situations. These contributions are not independent, as both electrostatic and hydrodynamic forces influence the suspension structure and hence both contributions to the stress. Since elasticity arises from nonhydrodynamic forces [Russel et al. (1989)], we consider only the electrostatic contribution to the stress, which is expressed

$$
\sigma_{x z}(t)=-\frac{1}{V} \sum_{i} z_{i} f_{x i}^{\text {total }}\left(\left\{\mathbf{r}_{j}(t)\right\}\right)
$$

where $\sigma_{x z}(t)$ is the dimensionless time-dependent shear stress acting in the $x$ direction on a plane normal to the $z$ direction, $f_{x i}^{\text {total }}\left(\left\{\mathbf{r}_{j}(t)\right\}\right)$ is the total dimensionless electrostatic plus short-range repulsive force acting on sphere $i$ in the $x$ direction, and $V$ is the dimensionless volume.

For linear oscillatory shear, the response can be represented by frequency-dependent storage $\left[G^{\prime}(\omega)\right]$ and loss $\left[G^{\prime \prime}(\omega)\right]$ components of the time-dependent shear stress,

$$
\frac{\sigma_{x z}(t)}{\gamma_{0}}=G_{e}+G^{\prime}(\omega) \sin \omega t+G^{\prime \prime}(\omega) \cos \omega t,
$$

where $G_{e}$ is the equilibrium modulus arising from a constant residual stress.

Obtaining $G^{\prime}(\omega)$ and $G^{\prime \prime}(\omega)$ from $\sigma_{x z}(t)$ is straightforward, but obtaining $\sigma_{x z}(t)$ can be computationally expensive, especially at low frequencies. For example, for simulation of a bidisperse suspension with sphere diameters $\sigma=1.0,0.1$, with $\omega=10^{-2}$ and $\Delta t=10^{-4}$, approximately $6.3 \times 10^{6}$ time steps are required to simulate one period, and several periods must be simulated before transients decay. Exploiting the interrelations among linear viscoelastic properties, $G^{\prime}(\omega)$ and $G^{\prime \prime}(\omega)$ can also be determined from the relaxation modulus $G(t)$ which can be obtained easily by stress relaxation after a small step strain.

$$
\begin{gathered}
G^{\prime}(\omega)=G_{e}+\omega \int_{0}^{\infty}\left[G(t)-G_{e}\right] \sin \omega t d t, \\
G^{\prime \prime}(\omega)=\omega \int_{0}^{\infty}\left[G(t)-G_{e}\right] \cos \omega t d t
\end{gathered}
$$

where the relaxation modulus is given by 


$$
G(t)=\frac{\sigma_{x z}(t)}{y_{0}^{\text {step }}}
$$

This method has the advantage that it saves a large amount of computing time compared to the direct simulation of oscillatory shear flow. For example, for the bidisperse system referred to above, a stress relaxation simulation requires on the order of only $10^{5}$ time steps, and a single simulation provides $G^{\prime}(\omega)$ and $G^{\prime \prime}(\omega)$ at all frequencies through Eqs. $(18)$ and $(19)$. However, $G^{\prime}(\omega)$ and $G^{\prime \prime}(\omega)$ can only be determined in this manner under linear deformation.

\section{Relaxation spectrum}

Linear viscoelastic responses are often discussed in terms of a relaxation spectrum $H(\tau)$, where $H(\tau) d \ln \tau$ is the contribution to the relaxation modulus from relaxation times between $\ln \tau$ and $\ln \tau+d \ln \tau$. The relaxation spectrum is related to the rheological properties described above by

$$
\begin{gathered}
G(t)=G_{e}+\int_{-\infty}^{\infty} H(\tau) e^{-t / \tau} d \ln \tau \\
G^{\prime}(\omega)=G_{e}+\int_{0}^{\infty} \frac{H(\tau) \omega^{2} \tau}{1+\omega^{2} \tau^{2}} d \tau
\end{gathered}
$$

and

$$
G^{\prime \prime}(\omega)=\int_{0}^{\infty} \frac{H(\tau) \omega}{1+\omega^{2} \tau^{2}} d \tau
$$

Investigating the relaxation spectrum provides two benefits. First, all linear viscoelastic properties can be obtained from $H(\tau)$. Second, relaxation events are identified more clearly from $H(\tau)$ than from $G(t)$ or $G^{\prime}(\omega)$ and $G^{\prime \prime}(\omega)$ because features in $H(\tau)$ tend to get smoothed out during the integration processes. However, relaxation spectra cannot be obtained directly by experiment or simulation, but must be extracted from viscoelastic properties [usually $G(t)$ or $G^{\prime}(\omega), G^{\prime \prime}(\omega)$ ] through the integral equations [Eqs. (21)(23)]. These are Fredholm integral equations of the first kind, and it is well known that solving for $H(\tau)$ is an ill-posed problem. Regularization [Honerkamp and Weese (1989)] and maximum entropy [Elster and Honerkamp (1991)] methods have been used to obtain solutions, but approximate solutions based on the differentiation of experimental data are also useful [Ferry (1980); Tschoegl (1989)]. Approximations suggested by Yasuda and Ninomiya [Tschoegl (1989)] are particularly useful for computer calculations:

$$
\begin{gathered}
H_{1 h}\left(\pi \beta_{1 h}\right)=\left.\frac{G(t)-G(h t)}{\ln h}\right|_{t=\tau}, \\
H_{2 h}\left(\pi \beta_{2 h}\right)=\left.\frac{h G(t)-(h+1) G(h t)+G\left(h^{2} t\right)}{(h-1) \ln \dot{h}}\right|_{t=\tau},
\end{gathered}
$$

and

$$
H_{3 h}\left(\pi \beta_{3 h}\right)=\left.\frac{h^{3} G(t)-h\left(h^{2}+h+1\right) G(h t)+\left(h^{2}+h+1\right) G\left(h^{2} t\right)-G\left(h^{3} t\right)}{\left(h^{2}-1\right)(h-1) \ln h}\right|_{t=\tau}
$$


$H_{1 h}, H_{2 h}$, and $H_{3 h}$ are increasing orders of approximation to the relaxation spectrum. $h$ is a constant, and the $\beta_{k h}$ 's are spectral shift factors which depend on $h$. In practice, a spacing of $\ln h=0.2$ is generally suggested, and in this case, $\beta_{k h}=0.787,1.263,1.550$ for $k=1,2$, and 3, respectively. In this study, Eq. (26) will be used to obtain the relaxation spectrum from the relaxation modulus. Once the relaxation spectrum has been determined, $G^{\prime}(\omega)$ and $G^{\prime \prime}(\omega)$ can be oblained from Eqs. (22) and (23), and compared to their direct calculation [Eqs. (18) and (19) or Eq. (17)] to assess the accuracy of the approximation. The effect of the order of approximation on the calculated relaxation spectrum will be discussed in the Results section.

\section{Partial rheological functions}

Before investigating the rheological response of various structures, we digress briefly to introduce a technique that is useful for identifying microscopic features that produce various rheological events. This method involves separating the stress calculation into contributions from various spheres or sphere interactions. Consider the shear stress $\sigma_{x z}$ [Eq. (16)] for suspension structures consisting of $N_{c}$ distinguishable clusters (see, for example, the structure depicted in Fig. 7). Let spheres $i=1,2,3 \ldots, k_{1}$ belong to cluster 1 , spheres $i=k_{1}+1, k_{1}+2, \ldots, k_{2}$ belong to cluster 2 , and so on. The shear stress can be rewritten as follows:

$$
\begin{gathered}
\sigma_{x z}=-\frac{1}{V}\left(\sum_{i=1}^{k_{1}} z_{i} f_{x i}^{\mathrm{total}}+\sum_{i=k_{1}+1}^{k_{2}} z_{i} f_{x i}^{\mathrm{total}}+\ldots\right) \\
=\sigma_{x z, 1}+\sigma_{x z, 2}+\ldots \\
=\sum_{j=1}^{N_{c}} \sigma_{x z, j}
\end{gathered}
$$

where

$$
\sigma_{x z, j}=\sum_{i=k_{j-1}+1}^{k_{j}} z_{i} f_{x i}^{\text {total }}
$$

represents the stress transmitted by the $j$ th cluster $\left(k_{0} \equiv 0\right)$. Note that the force on the $i$ th sphere may possess contributions from spheres in other clusters. This separation need not be restricted to grouping spheres by clusters; spheres can be grouped in any desired manner. We call the stress contribution $\sigma_{x z, j}$ the partial stress transmitted by the group of spheres designated by the subscript $j$. These partial stresses then produce partial moduli by substitution into Eqs. (17) or (20), and partial relaxation spectra through Eqs. (21)(23). Below, we show that this separation is useful for determining those combinations of spheres that give rise to specific relaxation events.

This analysis may be extended to situations where we not only want to determine the spheres responsible for specific relaxation events, but also the contributions to the force on each sphere controlling specific relaxations. Since the repulsive interaction between a sphere and an electrode only contributes to the $z$ component of the force on each sphere, $f_{x i}^{\text {total }}$ may be expressed as a series of summations over pair interactions only:

$$
f_{x i}^{\mathrm{total}}=\sum_{j \neq i}^{N} f_{x, i j}^{\mathrm{el}}+\sum_{j=1}^{N} f_{x, i j}^{\mathrm{el},}+\sum_{j \neq i}^{N} f_{x, i j}^{\mathrm{rep}} \text {. }
$$


Consider the situation where the spheres may be classified into two types, $\alpha$ and $\beta$ (e.g., spheres of two different sizes, $\sigma_{\alpha}$ and $\sigma_{\beta}$ ). The total force on sphere $i$ in the $x$ direction can be separated into a part arising from pair interactions with sphere type $\alpha$, and a part arising from interactions with sphere type $\beta$ :

$$
f_{x i}^{\text {total }}=f_{x i}^{\text {total, } \alpha}+f_{x i}^{\text {total }, \beta}
$$

where

$$
f_{x i}^{\mathrm{total}, \gamma}=\sum_{\substack{j \neq i \\ j \in \gamma}}^{N} f_{x, i j}^{\mathrm{el}}+\sum_{\substack{j=1 \\ j \in \gamma}}^{N} f_{x, i j}^{\mathrm{el},}+\sum_{\substack{j \neq i \\ j \in \gamma}}^{N} f_{x, i j}^{\mathrm{rep}}
$$

is the force on sphere $i$ due to all spheres of type $\gamma$. Since sphere $i$ itself may be of type $\alpha$ or $\beta$, substitution of this result into Eq. (16) produces three contributions to the total shear stress,

$$
\sigma_{x z}=\sigma_{x z}^{\alpha \alpha}+\sigma_{x z}^{\beta \beta}+\sigma_{x z}^{\alpha \beta}
$$

where

$$
\begin{gathered}
\sigma_{x z}^{\alpha \alpha}=-\frac{1}{V} \sum_{\substack{i=1 \\
i \in \alpha}}^{N} z_{i} f_{x i}^{\mathrm{total}, \alpha}, \\
\sigma_{x z}^{\beta \beta}=-\frac{1}{V} \sum_{\substack{i=1 \\
i \in \beta}}^{N} z_{i} f_{x i}^{\text {total }, \beta}
\end{gathered}
$$

and

$$
\sigma_{x z}^{\alpha \beta}=-\frac{1}{V_{i=1}} \sum_{\substack{i \in \alpha \\ i \in f_{i}}}^{N} z_{x i}^{\mathrm{total}, \beta}-\frac{1}{V_{i=1}} \sum_{\substack{i \in \beta \\ f_{x i}}}^{N} z_{x i}^{\mathrm{total}, \alpha}
$$

We also call the components $\sigma_{x z}^{\gamma \delta}$ partial stresses, and again, these give rise to partial moduli and partial relaxation spectra. The two types of partial quantities can be distinguished by the manner in which they are designated (i.e., with either subscripts or superscripts). We use these below to determine the source of relaxation events in bidisperse suspensions. This analysis can easily be extended to include more than two types of interactions, for example, by distinguishing between electrostatic and repulsive forces as well as distinguishing by sphere sizes.

\section{RESULTS}

\section{A. Monodisperse ER suspensions revisited}

In Paper I, a relaxation mechanism based on a transition from nonaffine to affine deformation of spheres within clusters was demonstrated. At small frequencies, deformation is completely determined by the displacement of spheres attached to the electrodeshydrodynamic resistance to sphere motion is insignificant at small deformation rates. As a result, the net electrostatic-plus-repulsive force on each sphere not attached to an electrode is zero at every instant. During the quasistatic deformation, each sphere will 


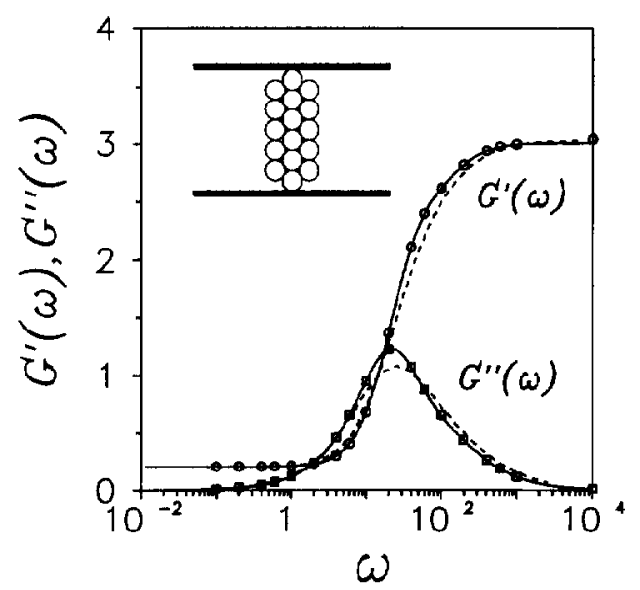

FIG. 2. Dynamic oscillatory response for a monodisperse structure consisting of manufactured 16-sphere clusters. The symbols represent direct simulation of oscillatory shear flow, the solid curves are moduli transformed from $G(t)$ (obtained from simulation of shear stress relaxation), and the dashed curves are moduli calculated from the relaxation spectrum (obtained from simulation of shear stress relaxation).

translate to satisfy this zero net electrostatic-plus-repulsive force. For arbitrary suspension structures, this will necessarily result in nonaffine motion for most spheres.

At large frequencies, the motion of spheres is controlled primarily by hydrodynamic forces. As clusters deform, spheres still feel a net electrostatic-plus-repulsive force attempting to move them into lower energy positions. However, during a half-period of oscillation, there is insufficient time for the spheres to move to these preferred positions. This time, proportional to $1 / \omega$, vanishes as $\omega \rightarrow \infty$. Hence, at large frequencies, each sphere displaces only in the direction of the ambient velocity, and motion is affine.

This transition from nonaffine to affine deformation provides a frequency-dependent dynamic structure. As the shear stress is simply a summation over position-dependent pair interactions, the stress and hence the moduli are also frequency dependent; this transition embodies the relaxation mechanism. This relaxation mechanism was identified by a simulation technique that employed simplified force expressions describing the motion of the spheres; hydrodynamic forces were represented by Stokes' drag and electrostatic interactions were treated in the point-dipole limit. The existence of a frequencydependent dynamic structure, however, transcends the level at which the forces are approximated. The electrostatic force on a sphere will, in general, possess a component in the direction of the hydrodynamic force as well as a component perpendicular to the hydrodynamic force. As the hydrodynamic force depends on the oscillation frequency, so will the resultant force on each sphere, providing a frequency-dependent dynamic structure and a mechanism for relaxation.

By examining the loss modulus for various suspension structures in Paper I, relaxation was found to occur over a fairly narrow range of frequencies. In this section, we consider this relaxation mechanism in more detail, and demonstrate that the observed relaxation depends sensitively on the relative positions of all spheres, and that a range of characteristic relaxation times exists.

Consider a suspension structure consisting of single-sphere-width strands spanning the electrode gap, with additional spheres placed in the triangular lattice positions along the side of the strands, as depicted in Fig. $2\left(N=16, L_{x}=10, L_{z}=6\right)$. The frequency- 


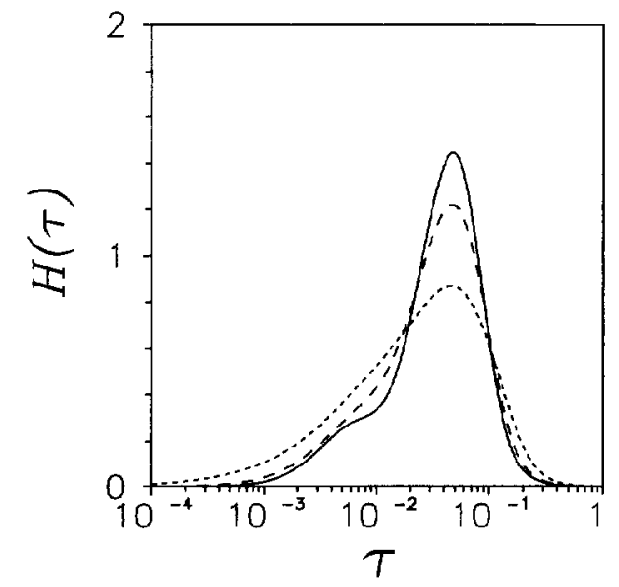

FIG. 3. Relaxation spectra for the structure presented in Fig. 2, obtained from simulation of stress relaxation. The short-dashed, long-dashed, and solid curves represent the first, second, and third order approximations of Yasuda and Ninomiya, respectively [Eqs. (24)-(26)].

dependent moduli for this structure are also presented in Fig. 2. To a first approximation, the relaxation is governed by a single relaxation time for this ordered monodisperse ER suspension structure. The loss modulus scales as $G^{\prime \prime}(\omega) \sim \omega$ at small frequencies and $G^{\prime \prime}(\omega) \sim \omega^{-1}$ at large frequencies, passing through a maximum near $\omega=25$. Near this frequency, $G^{\prime}(\omega)$ increases from a small frequency plateau $\left[G^{\prime}(0)\right]$ to a large frequency plateau $\left[G^{\prime}(\infty)\right]$. In Paper I, it was demonstrated that this response is typical of all monodisperse structures undergoing small amplitude deformation. Narrow dispersions are observed for structures confined to two-dimensional monolayers, as well as for threedimensional structures. Our preliminary results also indicate that the qualitative features presented here are unaltered by changing the range of the repulsive force, e.g., by increasing the value of $\kappa$ in Eqs. (5) and (6). As $\kappa$ is increased, the maximum in $G^{\prime \prime}(\omega)$ increases and shifts to larger frequencies, and $G^{\prime}(\infty)-G^{\prime}(0)$ increases as well. However, shapes of the curves are unchanged and the dispersion is still narrow, being characterized to a good approximation by a single relaxation time. The behavior of true hard-sphere suspensions has yet to be determined.

Results from the simulation of oscillatory shear are given together with the values obtained from the simulation of stress relaxation in Fig. 2. The symbols represent the dynamic moduli obtained directly from oscillatory simulations by Eq. (17); the solid curves represent values transformed from $G(t)$ using Eqs. (18) and (19); and the dashed curves represent approximate moduli obtained from $H(\tau)$ by Eqs. (22) and (23), wherc $H(\tau)$ was obtained from $G(t)$ by Eq. (26). The transformed moduli coincide with the direct simulation results, as expected, since Eqs. (18) and (19) are exact expressions. The approximate moduli deviate slightly, due to the approximations used in obtaining $H(\tau)$. However, the approximate values are in good agreement with the direct results, indicating that determining $H(\tau)$ from Eq. (26) captures the essential features of the rheological response in this situation.

More information can be inferred from the corresponding relaxation spectrum. $H(\tau)$ for the three different orders of approximation of Yasuda and Ninomiya [Eqs. (24)-(26)] are presented in Fig. 3. The highest order approximation produces a narrower distribution, while the lowest order approximation exhibits a smaller maximum, and does not 


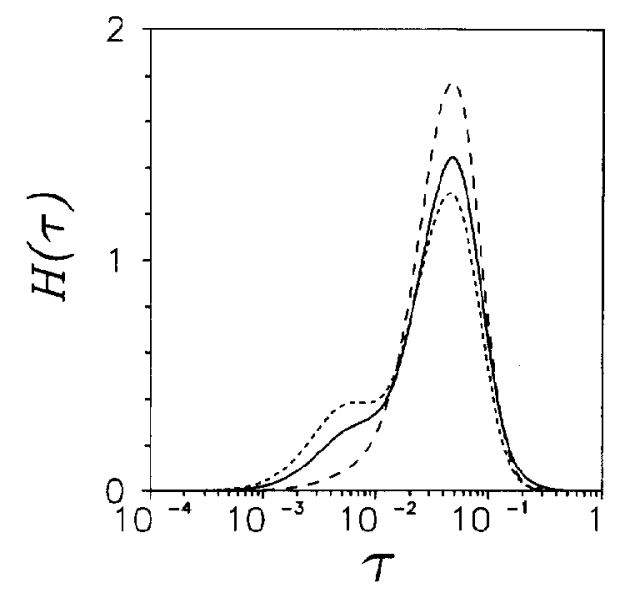

FIG. 4. Relaxation spectra for the structure presented in Fig. 2, for several electrode separations. Long-dashed curve, $L_{z}=5.96$; solid curve, $L_{z}=6.00$; short-dashed curve, $L_{z}=6.04$.

detect the shoulder at small times. However, the maximum occurs at the same position for all orders of approximation, and all of the curves are asymmetric. This figure shows that at least two relaxation events exist. Thus, the relaxation of this model structure is not as simple as might be presumed from the moduli alone. The feature producing these different relaxation times can be determined by perturbing the original structure, and by probing the response of simpler structures exhibiting similar behavior.

The structure depicted in Fig. 2 is stretched along the $z$ direction. When the electrodes are removed and the structure is allowed to relax, the distance between the top and bottom spheres, $z_{\text {top }}-z_{\text {bottom }}$, decreases from 5.017 to 5.005 . When the original structure is perturbed, it will rearrange to some extent, in order to release some of the energy stored in the stretched configuration. To examine the impact of the degree of stretching on the rheological response, stress relaxation simulations were performed on perturbations of this structure. The electrodes were displaced by small amounts in the $z$ direction, the simulation was run under no flow until all motion ceased, and then stress relaxation was simulated. Resulting relaxation spectra are presented in Fig. 4 for electrode separations $L_{z}=5.96,6.00$, and 6.04 . The magnitude of the small relaxation time shoulder decreases and the magnitude of the main peak increases as the gap is decreased. At a separation of $L_{z}=5.96$, the shoulder has almost entirely disappeared, although the relaxation spectrum is still slightly skewed to small relaxation times, and the magnitude of the major peak has increased by $22 \%$ compared to its value for $L_{z}=6.00$. The positions of the peaks on the time axis, however, do not appear to change as the gap is changed. Thus, for this configuration, stretching appears to influence only the magnitudes of the peaks in $H(\tau)$.

The relaxation mechanisms can be probed further by examining the relaxation spectrum of the three-sphere cluster presented in Fig. $5\left(N=3, L_{x}=10, L_{z}=2\right)$. This structure possesses features contained in the structure illustrated in Fig. 2; a chain of two spheres connecting the electrodes (spheres 1 and 2), with an additional sphere (sphere 3) placed in a triangular lattice position. The solid curve in Fig. 5 represents the total relaxation spectrum, calculated from the forces on all spheres obtained from the simulation of stress relaxation. This spectrum is similar to the spectrum presented in Fig. 3; a main peak at $\tau \approx 0.07$ and a shoulder at $\tau \approx 0.007$. The long-dashed curve represents the 


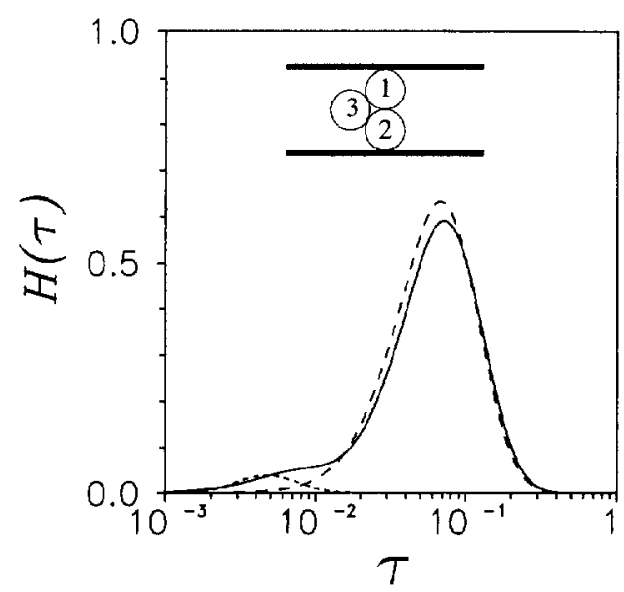

FIG. 5. Relaxation spectra for a monodisperse structure consisting of manufactured 3-sphere clusters. The solid curve is the total spectrum obtained from simulation of stress relaxation. The long-dashed curve is the partial spectrum $H_{3}(\tau)$ obtained from the perturbation solution for shear stress relaxation. The short-dashed curve is the partial spectrum $H_{1,2}(\tau)$ obtained from simulation of shear stress relaxation.

partial relaxation spectrum obtained from the partial modulus $G_{3}(t)$, where the trajectories were obtained from the perturbation solution for stress relaxation (see the Appendix for details on the perturbation analysis for this three-sphere configuration). Only a single peak is observed, approximately of the same magnitude, and occurring at the same position on the time axis, as the major peak in the solid curve. In the perturbation analysis, spheres 1 and 2 are constrained to fixed positions at the electrodes during relaxation, removing the opportunity for the cluster to relax stretching energy; hence, relaxation arises only from the motion of sphere 3 . Since the shoulder at short times is absent in the long-dashed curve, we conclude that the shoulder arises from relaxation of stretching energy. The short-dashed curve represents the partial spectrum obtained from the partial modulus $G_{1,2}(t)$ obtained from the simulation of stress relaxation. In this case, only a small peak is observed, approximately of the same magnitude and occurring at approximately the same position on the time axis as the shoulder in the solid curve. This is expected, since the sticking condition employed in the simulation allows motion of spheres 1 and 2 only in the direction normal to the electrodes; hence, relaxation of spheres 1 and 2 can only relieve energy stored by stretching in the $z$ direction.

The relaxation spectrum for another manufactured monodisperse structure is presented in Fig. $6\left(N=13, L_{x}=10, L_{z}=7.928\right)$. This spectrum possesses only a single peak, located at approximately the same position on the time axis as the shoulder in Fig. 3, but of larger magnitude. When the electrodes are removed, the distance between the top and bottom spheres, $z_{\text {top }}-z_{\text {bottom }}$, decreases from 6.943 to 6.901 . Hence stretching energy is also stored in this cluster, which is expected to contribute to the relaxation. To investigate the influence of stretching energy relaxation on the relaxation spectrum, stress relaxation was simulated again for this structure perturbed by slightly displacing the electrodes in the $z$ direction. The resulting relaxation spectra are also presented in Fig. 6 . In contrast to the results depicted in Fig. 4, the spectra in Fig. 6 appear to be well characterized by a single peak for all electrode separations, and displacing the electrodes influences both the magnitude and position of the main peak. This implies that the influence of stretching 


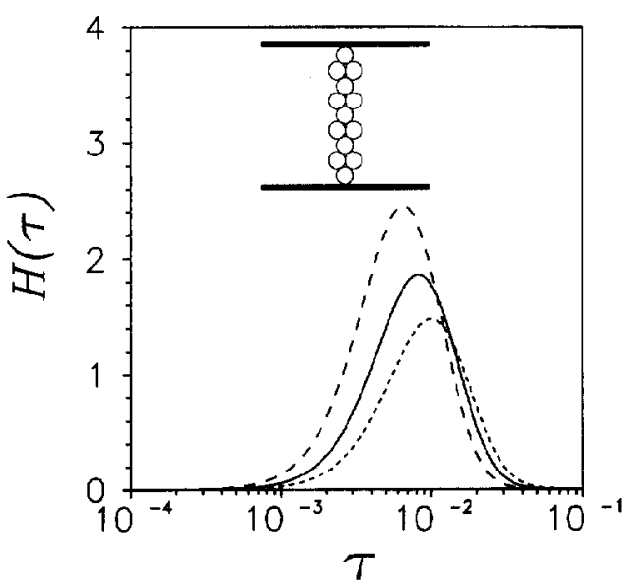

FIG. 6. Relaxation spectra for a monodisperse structure consisting of manufactured 13-sphere clusters, for several electrode separations. The solid curve is the spectrum for $L_{z}=7.928$, the short-dashed curve is for $L_{z}=7.948$, and the long-dashed curve is for $L_{z}=7.908$.

may not be simply assigned to a specific effect on specific peaks in $H(\tau)$; its influence on the response, as well as the response in general, depends on the relative positions of all the spheres.

As a final example of the response of a monodisperse structure, the moduli and relaxation spectra obtained from the simulation of stress relaxation for a structure formed from a random configuration are presented in Figs. 7 and 8, respectively $\left(N=51, L_{x}=10, L_{z}=10\right)$. This structure contains sphere configurations similar to those present in the structures investigated above as well as others. The shapes of the moduli curves are similar to those of the manufactured structures of Fig. 2 . The only significant differences are that $G^{\prime \prime}(\omega)$ is slightly broader, and is shifted to larger frequen-

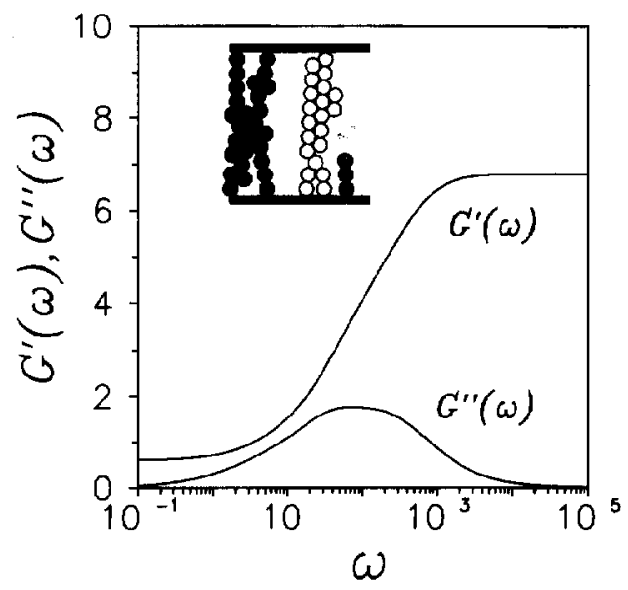

F1G. 7. Dynamic moduli as a function of frequency for a monodisperse structure formed from a random distribution of spheres, obtained from simulation of shear stress relaxation. 


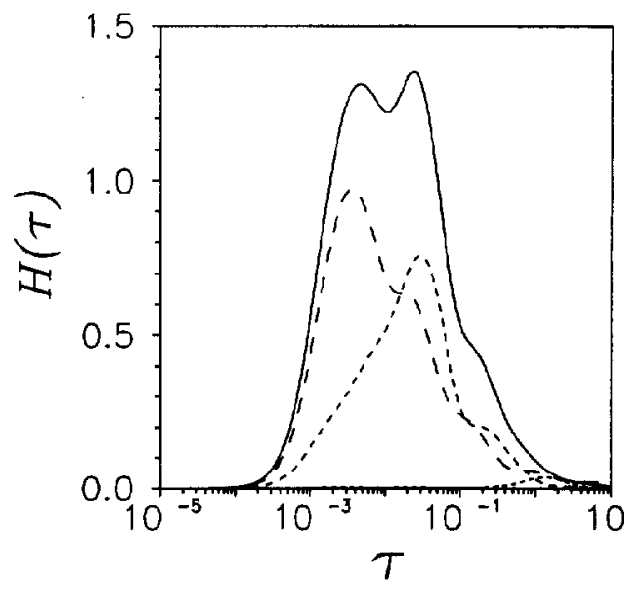

FIG. 8. Relaxation spectra for the structure presented in Fig. 7 , obtained from simulation of shear stress relaxation. The solid curve is the total relaxation spectrum. The long-dashed curve is the partial spectrum of the cluster on the left (Fig. 7), indicated by solid spheres. The medium-dashed curve is the partial spectrum of the cluster in the center, indicated by outlined spheres. The short-dashed curve is the partial spectrum of the 3-sphere cluster on the right.

cies. The width at half of the maximum of the $G^{\prime \prime}(\omega)$ peak is greater than two decades in this case, compared to less than two decades for the former case. The corresponding relaxation spectra are shown in Fig. 8. The solid curve represents the total spectrum determined from the relaxation modulus including contributions from all spheres. Four relaxation events are apparent from this curve; peaks or shoulders occur at $\tau \approx 0.005$, $0.03,0.2$, and 5 . The long-dashed curve represents the partial spectrum for the 27 -sphere cluster on the left, indicated by solid black spheres. This spectrum exhibits five relaxation events at times $\tau \approx 0.004,0.02,0.13,1$, and 6 . The medium-dashed curve represents the partial spectrum for the 21 sphere cluster in the center, indicated by outlined white spheres. This spectrum also appears to exhibit five relaxation events at times $\tau \approx 0.0015$, $0.006,0.03,0.2$, and 3 . The short-dashed curve represents the partial spectrum for the three-sphere cluster on the right, indicated by the outlined gray spheres. This spectrum exhibits a single relaxation event at $\tau \approx 1.3$ [McLeish et al. (1991) have described the relaxation of "free strings"- - single-sphere-width strands attached to at most oneelectrode. These structures are likely to influence the response at small concentrations, and can produce large relaxation times. In this paper, we focus on the response of concentrated suspensions where such structures are not likely to influence the response.] The partial spectra of the separate clusters are no less complex than the total spectrum.

In principle, one could evaluate the partial spectra for various sphere combinations, in order to determine the structural entities that produce various relaxation events. However, this procedure will become tedious as the number of spheres is increased. The purpose of analyzing the response of simple structures in this manner is to demonstrate two features. First, the response depends, in a nontrivial way, on the relative positions of all of the spheres. As illustrated in Figs. 4 and 6, the responses of even simple structures vary, and small perturbations influence the responses of different structures in different ways. Second, the response of even simple structures is often not characterized by a single relaxation time. For the monodisperse structures considered here, we find that the breadth of dispersion, characterized by the width at half of the maximum of $G^{\prime \prime}(\omega)$ or $H(\tau)$, is 


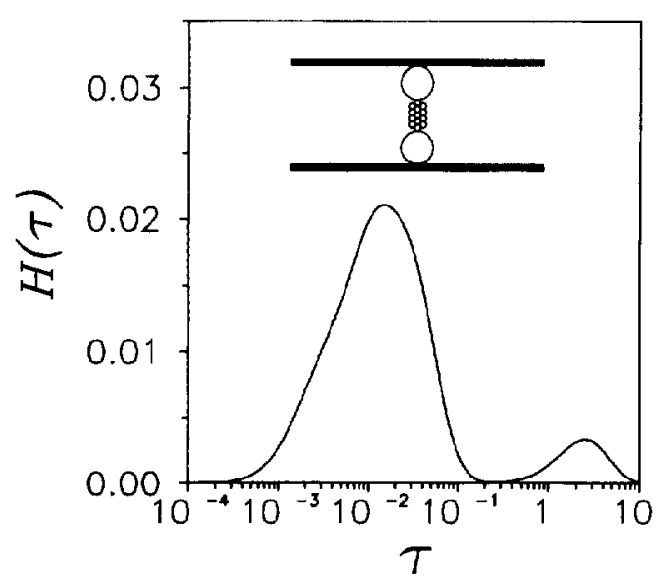

FIG. 9. Relaxation spectrum for a bidisperse structure consisting of manufactured 15-sphere clusters $\left(\sigma_{\text {smal }} / \sigma_{\text {large }}=0.2\right)$, obtained from simulation of shear stress relaxation.

approximately two decades, with relaxation times contained largely in the region $10^{-3}<\tau<10^{-1}$. In the following section, we consider how polydispersity influences the breadth of dispersion.

\section{B. The influence of polydispersity}

Simulating the response of polydisperse suspensions is straightforward; spheres of various sizes are simply placed in the simulation cell, and size dependent interactions are evaluated as described in Sec. II. The electrostatic interaction between spheres of different diameters is essentially the same as that between two spheres of the same size (in the point-dipole limit). The dipolar nature of the interaction is maintained for all size ratios $\lambda_{i j}=\sigma_{i} / \sigma_{j}$, but the magnitude of the interaction is a sensitive function of this diameter ratio. In fact, in the limit as $\lambda_{i j} \rightarrow 0$ or $\infty$, the magnitude of the interaction vanishes. Considering the role of electrostatic interactions in the nonaffine-affine transition that controls the relaxation process, we expect the presence of spheres of different size to produce relaxation events over a broader range of relaxation times than observed for monodisperse suspensions.

Consider the manufactured, bidisperse structure illustrated in Fig. 9. Two large spheres $(\sigma=1)$ are placed at the electrodes, and thirteen small spheres $(\sigma=0.2)$ are placed between the large spheres, forming a cluster similar in shape to the cluster illustrated in Fig. 2. The relaxation spectrum for this bidisperse structure is also presented in Fig. 9. There are two distinct peaks at $\tau \approx 0.015$ and $\tau \approx 2.5$. The features producing each peak can be identified from the partial loss moduli, which are presented in Fig. 10. The solid curve is the total loss modulus, the long-dashed curve is the partial modulus $G^{S S^{\prime \prime}}(\omega)$ determined by calculating the stress arising from interactions between small spheres, and the short-dashed curve is the partial modulus $G^{S L^{\prime \prime}}(\omega)$ determined by calculating the shear stress arising from interactions between small and large spheres. It is apparent that the small peak at $\omega \approx 0.4$ arises from the interactions between small and large spheres and the peak at $\omega \approx 67$ comes primarily from the interactions between small spheres. Referring to the relaxation spectrum (Fig. 9), it is evident that the peak in $H(\tau)$ at 


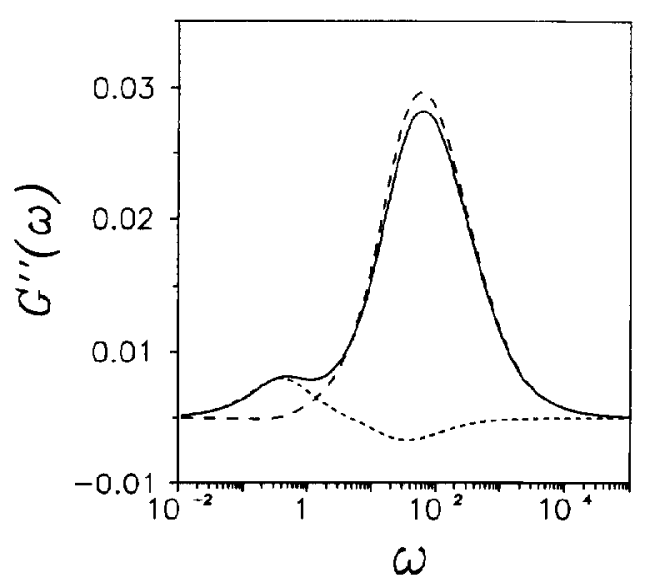

FIG. 10. Loss moduli for the structure presented in Fig. 9, obtained from simulation of shear stress relaxation. The solid curve is the total loss modulus. The long-dashed curve is the partial loss modulus $G^{S S^{\prime \prime}}(w)$. The short-dashed curve is the partial loss modulus $G^{S L^{\prime \prime}}(\omega)$.

$\tau \approx 0.015$ arises from the interaction between small spheres, while the peak at $\tau \approx 2.5$ is due to the interaction between large and small spheres.

It is apparent that the presence of spheres of different size offers a mechanism for dispersion broadening, but as discussed in the previous section, the response also depends on the relative positions of all spheres. To probe the influence of relative sphere diameters as well as the relative sphere positions on the response, several bidisperse structures formed from random configurations were investigated. The structures and relaxation spectra obtained from stress relaxation simulations are presented in Figs. 11-14. These structures possess a variety of diameter ratios ranging from $0.2 \leqslant \lambda_{i j} \leqslant 1$. In each figure, the total relaxation spectra are plotted along with the partial spectra $H^{S S}(\tau), H^{S L}(\tau)$, and

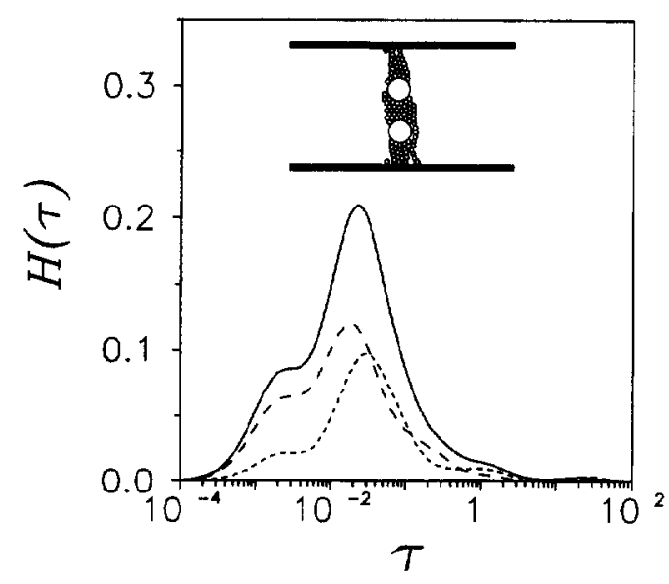

FIG. 11. Relaxation spectra for a bidisperse structure formed from a random distribution of spheres $\left(\sigma_{\text {small }} / \sigma_{\text {large }}=0.2\right)$, obtained from simulation of shear stress relaxation. The solid curve is the total spectrum. The long-dashed curve is the partial spectrum $H^{S S}(\tau)$. The short-dashed curve is the partial spectrum $H^{S L}(\tau)$. 


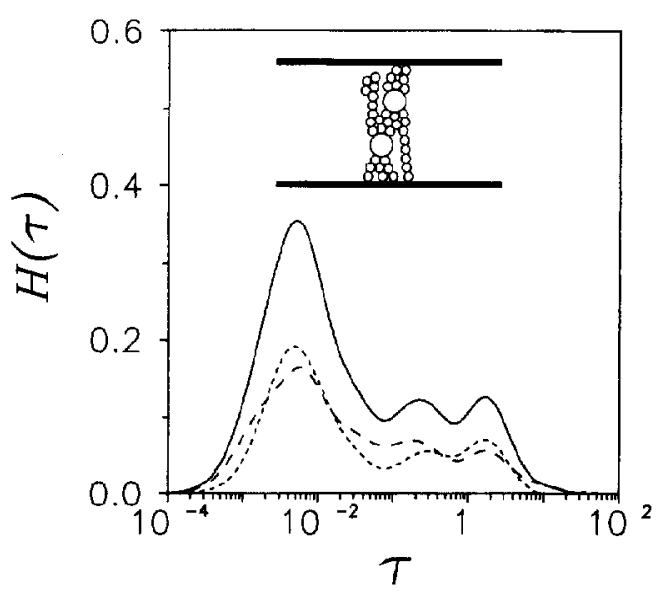

FIG. 12. Relaxation spectra for a bidisperse structure formed from a random distribution of spheres $\left(\sigma_{\text {small }} / \sigma_{\text {large }}=0.4\right.$ ), obtained from simulation of shear stress relaxation. The solid curve is the total spectrum. The long-dashed curve is the partial spectrum $H^{S S}(\tau)$. The short-dashed curve is the partial spectrum $H^{S L}(\tau)$.

in Fig. 14, $H^{L L}(\tau)$. Common features in each of these responses are: (1) the total spectra possess several discernible peaks and shoulders, (2) the partial spectra tend to demonstrate as many events as the total spectra, and (3) most significantly, with the exception of Fig. 12, the spectra do not display any increased broadening relative to those observed for random monodisperse structures, the spectra being contained largely in the region $10^{-3} \leqslant \tau \leqslant 10^{-1}$ [as characterized by $H(\tau) \geqslant \frac{1}{2} H\left(\tau_{\max }\right)$ ]. The relatively broad spectrum in Fig. 12 arises from the large-time relaxation of the pendant cluster of spheres on the upper left side of the structure; when this group of spheres is removed (Fig. 13) the

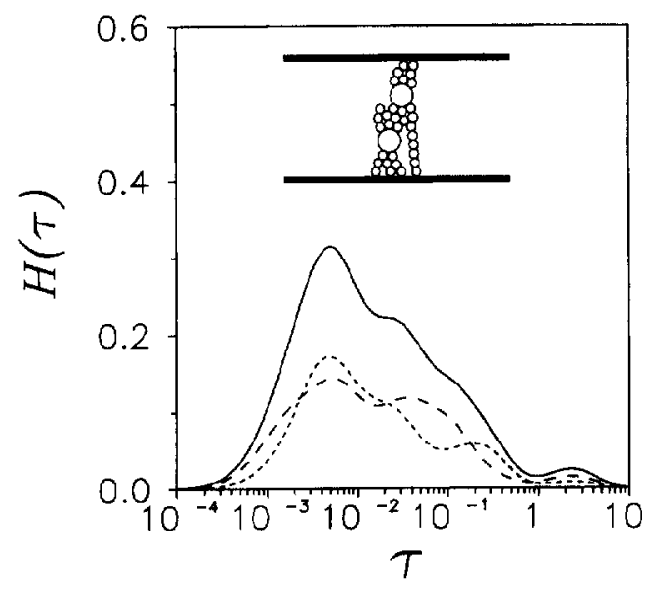

FIG. 13. Relaxation spectra for a bidisperse structure obtained by removing the pendant cluster of 6 spheres on the upper left side of the cluster depicted in Fig. 12, followed by simulation under no flow. $\left(\sigma_{\text {smalf }} / \sigma_{\text {large }}=0.4\right)$. The solid curve is the total spectrum. The long-dashed curve is the partial spectrum $H^{S S}(\tau)$. The short-dashed curve is the partial spectrum $H^{S L}(\tau)$. 


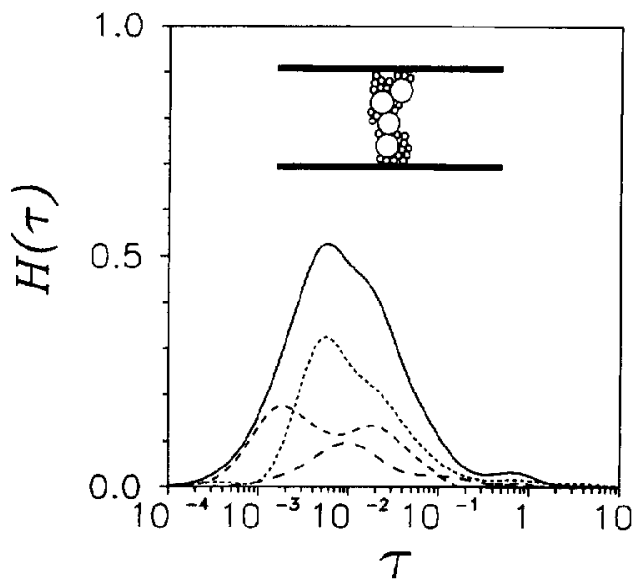

FIG. 14. Relaxation spectra for a bidisperse structure formed from a random distribution of spheres $\left(\sigma_{\text {small }} / \sigma_{\text {large }}=0.3\right.$ ), obtained from simulation of shear stress relaxation. The solid curve is the total spectrum. The long-dashed curve is the partial spectrum $H^{S S}(\tau)$. The medium-dashed curve is the partial spectrum $H^{L L}(\tau)$. The short-dashed curve is the partial spectrum $H^{S L}(\tau)$.

response again exhibits a fairly narrow dispersion. The broader dispersion in Fig. 12 is due to a specific configuration of spheres whose effect could be realized in a monodisperse structure. Hence the anticipated effect of polydispersity broadening the dispersion is not realized in these structures.

The role of relative sphere diameters on the breadth of dispersion can be probed further by investigating the three-sphere structure illustrated in Fig. 15. As discussed above, this simple structure exhibits the nonaffine-affine transition. By decreasing the

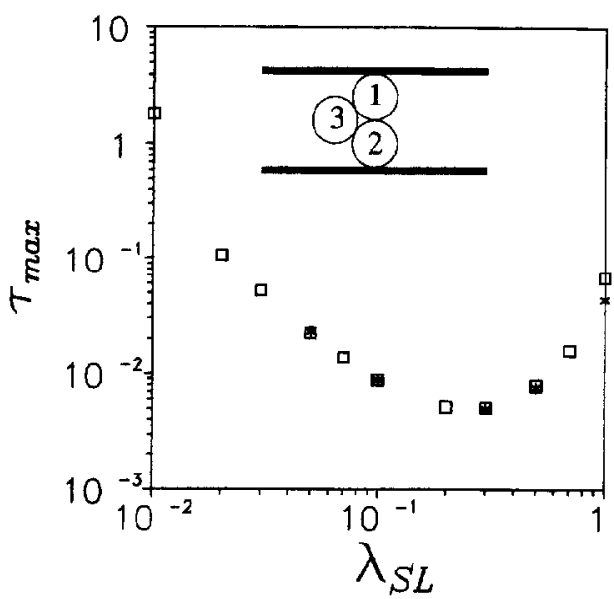

FIG. 15. Location of peak in the relaxation spectrum as a function of $\lambda_{S L}\left(=\sigma_{3}\right)$. The open squares were obtained from the perturbation solution of shear stress relaxation for the 3-sphere cluster, and the asterisks were obtained from simulation of shear stress relaxation for the 16-sphere cluster depicted in Fig. 2 (with the spheres in the side chains replaced by spheres with $\sigma=\lambda_{S L}$ ). 


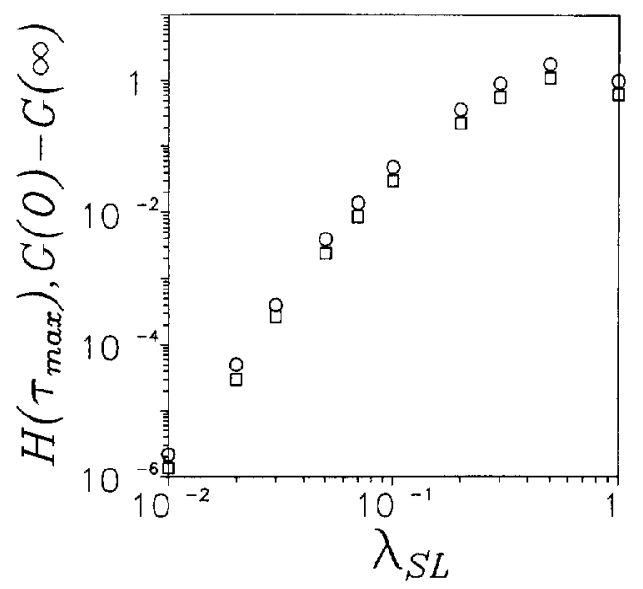

FIG. 16. $H\left(\tau_{\max }\right)$ (open squares) and $G(0)-G(\infty)$ (open circles) as a function of $\lambda_{S L}$, obtained from the perturbation solution of shear stress relaxation for the 3-sphere cluster depicted in Fig. 15.

diameter of sphere $3\left(\sigma_{3}=\lambda_{S L}\right)$ while maintaining contact with the other two spheres $\left(\sigma_{1}=\sigma_{2}=1\right)$, the effect of polydispersity can be investigated. The relaxation spectrum was determined for various values of $\lambda_{S L}$ using the regular perturbation solution for stress relaxation. In each case, the spectrum consisted of a single symmetric peak. The dependence of the peak position, $\tau_{\max }$ on $\lambda_{S L}$ is presented in Fig. 15. Also included in this figure are the peak positions of the relaxation spectrum for the structure illustrated in Fig. 2, where the spheres in the side chains were replaced with spheres of diameter $\sigma=\lambda_{S L}$. The two sets of data coincide very well except when $\lambda$ approaches unity, where interactions between side spheres, and between side spheres and their images, become sufficiently strong to alter the response of the 16-sphere cluster (in fact, for $0.6<\lambda_{S L}<0.9$, the manufactured structure is unstable and rearranges significantly). The agreement between the responses for $\lambda_{S L} \leqslant 0.5$ confirms that the three-sphere structure represents a useful simplification for probing polydispersity. This is particularly significant, since the time step required for simulation of stress relaxation is proportional to $\lambda_{S L}$, prohibiting simulations for small $\lambda_{S L}$.

As $\lambda_{S L}$ decreases, $\tau_{\max }$ decreases by approximately one decade, reaches a minimum near $\lambda_{S L}=0.3$, and then increases. Since $\tau_{\max }$ increases as $\lambda_{S L}$ decreases (for small $\lambda_{S L}$ ), we expect relaxation events to appear at large relaxation times for suspensions containing spheres very different in size. However, as $\lambda_{S L}$ decreases, the extent of relaxation, defined as either $G(0)-G(\infty)$ or $H\left(\tau_{\max }\right)$, also decreases. In Fig. 16, $G(0)$ $-G(\infty)$ and $H\left(\tau_{\max }\right)$, are plotted against $\lambda_{S L}$ (obtained from the perturbation solution of stress relaxation for the three-sphere structure). Both possess a maximum near $\lambda_{S L}=0.7$ and decrease rapidly as $\lambda$ decreases. When $\lambda=0.025, H_{\max }$ and $G(0)-G(\infty)$ are just $0.03 \%$ of their values at $\lambda=1$. As $\lambda_{S L}$ decreases, the relaxation moves to larger relaxation times (smaller frequencies), but the extent of relaxation decreases. As $\lambda_{S L} \rightarrow 0$, the storage modulus approaches a constant value, independent of frequency and equal to that for spheres 1 and 2 alone.

This behavior is consistent with description of the nonaffine-affine transition mechanism for relaxation. For small $\lambda_{S L}$, the relaxation is shifted to smaller frequencies (larger relaxation times) because the electrostatic force driving nonaffine motion is smaller. The 


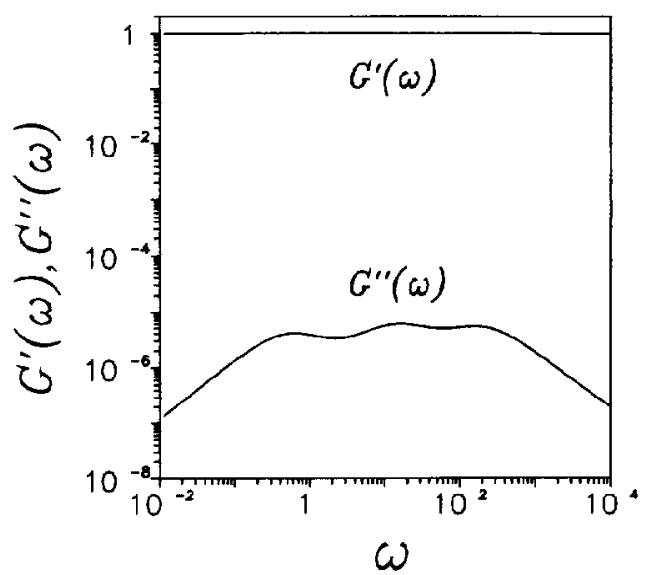

FIG. 17. Storage and loss moduli as a function of frequency for a model structure composed 3-sphere clusters. Each periodic "box" consists of one cluster with $\lambda_{S L}=1$, one cluster with $\lambda_{S L}=0.25$, and $3 \times 10^{5}$ clusters with $\lambda_{S L}=0.01$.

small and large frequency dynamic structures differ only through relatively weak forces, producing a smaller extent of relaxation.

To further demonstrate this point, consider a two-dimensional suspension structure consisting of a distribution of the three-sphere clusters, each containing a small sphere with an arbitrary diameter, and separated from one another by $\Delta x=10$. The loss modulus for such a structure can be represented as

$$
G^{\prime \prime}(\omega)=\sum_{i=1}^{N_{c}} f\left(\lambda_{S L, i}\right) \int_{0}^{\infty} \frac{H\left(\tau, \lambda_{S L, i}\right) \omega}{1+\omega^{2} \tau^{2}} d \tau,
$$

where $f\left(\lambda_{S L, i}\right)$ represents the fraction of three-sphere clusters with $\lambda_{S L}=\lambda_{S L, i}$ and $H\left(\tau ; \lambda_{S L, i}\right)$ represents the relaxation spectrum for these clusters. We further assume that the periodic image of the structure consists of one cluster with $\lambda_{S L}=1$, one cluster with $\lambda_{S L}=0.25$, and $2 f /(1-f)$ clusters with $\lambda_{S L}=10^{-2}$. We now wish to determine the value of $f$ that produces a "broad" dispersion, defined as the width at half of the maximum of $G^{\prime \prime}(\omega)$ equal to three decades. Assuming that the response of each individual cluster is unchanged by the presence of the others, we find that the required value of $f$ is 0.999994 (the dynamic moduli for this structure are presented in Fig. 17). Hence, for every cluster with $\lambda_{S L}=1$, approximately $3 \times 10^{5}$ clusters with $\lambda_{S L}=10^{-2}$ are required to observe a "broad" dispersion. Furthermore, since this suspension structure is dominated by the large number of small $\lambda_{S L}$ clusters, the extent of the relaxation $\left[G^{\prime}(\infty)-G^{\prime}(0)\right]$ is insignificant.

The structure employed in this example is clearly oversimplified. In fact, it is unlikely that mixing spheres of the proper size in the correct proportions could ever achieve the desired structure. However, this example demonstrates that in order to observe a significantly broadened dispersion arising from only polydispersity, a very peculiar suspension structure is required, and that the corresponding extent of relaxation is insignificant for such a situation. Hence, for typical polydisperse suspensions, we find that the dispersions observed under linear deformation will be insignificantly broader than those observed for monodisperse suspensions. 


\section{CONCLUSION}

The major results of this work are summarized as follows:

(1) Polydispersity, although capable of producing relaxation events over an arbitrarily broad range of relaxation times, does not significantly broaden dispersion; features that produce events at very large relaxation times do not contribute significantly to the extent of relaxation.

(2) For even some simple, monodisperse suspension structures, relaxation is not characterized by a single relaxation time. The observed response depends in a nontrivial way on the relative positions of all of the particles. For structures investigated in this study, relaxation events are found to be largely contained in the range of dimensionless relaxation times $10^{-3} \leqslant \tau \leqslant 10^{-1}$.

(3) Simulation of shear stress relaxation offers considerable computational savings over simulated oscillatory shear flow, while the simulation method itself differs only slightly from the methods employed previously.

(4) Perturbation solutions to the microscopic model for ER behavior offer additional advantages to studying the transient response. The time-dependent particle trajectories and rheological functions are determined directly, without the need for timeconsuming simulations (except perhaps to determine the equilibrium structure). However, extending these methods to nonlinear deformation will be tedious.

(5) Partial rheological functions are useful for determining causes of relaxation events. The definitions of these used in this paper may be easily extended to probe the influence of other features on the rheological response, such as distinguishing between the roles of attractive and repulsive forces, or other forces that may be present in actual ER suspensions.

This study has considered the linear response of monodisperse and polydisperse ER suspensions conforming to the electrostatic polarization model. Electrostatic forces were treated in the point-dipole limit, neglecting higher order electrostatic multipoles and multibody interactions, while hydrodynamic forces were treated as Stokes' drag, neglecting hydrodynamic interactions. We expect that incorporating more accurate electrostatic and hydrodynamic forces will alter the magnitudes of the rheological functions, as well as shift events in the time/frequency domains, but should not alter the qualitative information provided in this study. The relaxation mechanism arising from the frequencydependent dynamic structure, as well as the conclusion that features that produce relaxation events over broad ranges of relaxation times (i.e., polydispersity) do not broaden the dispersion, transcend the level at which forces are calculated. Perhaps a more severe restriction of this model is that we have neglected other phenomena that may be relevant to some ER suspensions employed in applications. Such phenomena include conduction (electronic and ionic), gravity, colloidal interactions, and Brownian motion.

We have not considered how polydispersity influences the magnitude of the rheological response, although the figures presented in this paper suggest that the impact is only minor at moderate concentrations. Such a simulation study would require large numbers of particles, beyond the scope of the present investigation, and would clearly be more amenable to experimental investigation. In contrast, the simulation and perturbation studies presented here are capable of probing many decades in (dimensionless) frequency, as opposed to the relatively limited frequency ranges of most experimental techniques.

Our modeling efforts to this point have considered monodisperse and polydisperse structures subjected to small amplitude (linear) deformation. These studies have yet to identify mechanisms that produce significantly broad dispersions, as are commonly observed experimentally (Paper I). Other features must be included in the simplified model 
presented in this paper in order to produce broad dispersions. Evidence for such possible features currently exists, and is discussed briefly below.

McLeish et al. (1991) modeled the dynamic response of ER suspensions by considering the suspension structure to be composed of single-sphere-width chains. A fraction of these chains extend from one electrode to the other, while the remaining chains are "frce," attached to, at most, one electrode. The authors demonstrated that the free strands can relax with long relaxation times, and thus contribute significantly to dispersion broadening. As pointed out in Paper I, free strands are numerous at small particle concentrations, but are less likely to exist in significant number at moderate to large concentrations typical for ER suspensions. As a result, we expect the influence of free chains to be insignificant above some critical concentration. However, the concept of free strings and their effect on the relaxation behavior may be extended to include pendant chainsclusters attached to precolating structures but not directly attached to an electrode, as depicted in Fig. 12. Indeed, Figs. 12 and 13 illustrate that such pendant clusters do produce relaxation events at large times and hence contribute to dispersion broadening. The impact on dispersion broadening at particle concentrations typical for ER suspensions remains to be determined.

Another possible broadening mechanism lies in the slip condition at the electrodes. To this point, we have treated spheres within a small distance of the electrode to be constrained to follow the lateral displacement of the electrodes. Preliminary simulations show that when this condition is removed, relaxation is broadened significantly. This arises from the slow relaxation (rotation) of entire clusters, in a manner similar to the relaxation of free chains described by McLeish et al. (1991). As discussed in Sec. II, we have also occasionally observed strain-induced slip for simulations at large deformations $\left(\gamma_{0}>1\right)$, where neighboring spheres pull constrained spheres away from the electrodes, allowing them to move parallel to the electrode surface. We have not observed such behavior for small amplitude, linear deformation $\left(\gamma_{0} \leqslant 10^{-4}\right)$.

Experimental evidence for the behavior of particles at electrodes indicates that particles do tend to stick for small to moderate deformations. Klingenberg and Zukoski (1990) found that for suspensions of silica spheres in corn oil ( $2 \%$ by volume), the spheres at the electrodes remained attached for moderate strain amplitudes $\left(\gamma_{0} \lesssim 0.2\right)$. Sprecher et al. (1990) measured the force required to shear a column of 3-5 particles spanning the electrode gap. They found that the particles stuck to the electrodes for small deformations, but began to slip at moderate deformations $[\gamma \sim \mathcal{O}(0.1)]$; to prevent slipping, the authors fabricated a notch on the electrode to hold particles in place [Conrad (1994)]. Hence, although it is clear that the behavior of particles at the electrode surface can have a pronounced effect on rheological properties, the variables influencing stick/ slip behavior (e.g., strain amplitude, surface roughness, particle size, etc.) and the corresponding relationships have yet to be determined. In particular, it is unclear whether or not slip contributes to relaxation in the linear regime or only arises under nonlinear deformation. The latter is addressed further below.

Nonlinear deformation has been shown to produce significantly different responses than those predicted here for linear deformation. Otsubo et al. (1992) observed that rheological responses for silica/silicone oil suspensions at small strain amplitudes $\left(\gamma_{0} \sim 0.01\right)$ were linear and were well represented by a single relaxation time. However, for larger strain amplitudes, the moduli increased slowly with frequency over a wide frequency range, indicating broader dispersion. The authors attributed this behavior to nonlinear deformation associated with yielding of the chain structure.

Parthasarathy et al. (1993) observed similar trends using the idealized model and computer simulation technique described here, as well as for experimental studies with 
alumina particles in poly(dimethylsiloxane). Computer simulations suggested that nonlinearity does not arise initially from macroscopic rupture of the particulate columns, but rather from slight rearrangements within clusters. Critical strains making the onset of nonlinearity were found to agree well with those obtained experimentally; these strains were found to be functions of both the electric field strength and oscillation frequency, with critical strains increasing as $\omega / E_{0}^{2}$ increased. Experimental strain sweep experiments at small oscillation frequencies demonstrated that the suspensions do exhibit a linear viscoelastic response for $\gamma_{0} \leqslant 10^{-3}$, while both the storage and loss moduli (obtained from the fundamental harmonic of the Fourier transform of the shear stress) decreased with increased strain amplitude $\left(\gamma_{0} \leqslant 0.02\right)$. The critical strain increased with frequency, leading to broader dispersions for nonlinear deformation.

Thus nonlinear deformation, associated with some level of structural rearrangement, appears to be a likely mechanism for significant dispersion broadening. It remains to be determined how rheological properties depend on strain amplitude, in particular, how the character of the response changes as one passes from the linear limit to a regime dominated by slight rearrangements in the suspension structure, and then on to larger scale changes that may include chain rupture or strain-induced slip at the electrodes. As a final note, although we find that polydispersity does not significantly alter the response observed for monodisperse suspensions under linear deformation, polydispersity may play a more significant role in determining the critical strain and the response under nonlinear deformation.

\section{ACKNOWLEDGMENTS}

This work was supported in part by an Air Force Engineering Research Initiation Grant (RI-B-92-07).

\section{APPENDIX}

In this Appendix, we outline a regular perturbation solution to the equations of motion presented in Sec. II. Linearized equations are developed for arbitrary structures and deformations, which are then solved in detail for a particular configuration of spheres undergoing shear stress relaxation.

It is convenient to separate the total electrostatic and repulsive force on sphere $i$ into the sum of the pair forces that depend on only $\mathbf{r}_{i}$ and $\mathbf{r}_{j}$ and an external force which depends on $L_{z}$ as well as $\mathbf{r}_{i}$ and $\mathbf{r}_{j}$ :

$$
\mathbf{f}_{i}^{\mathrm{tot}}=\sum_{j \neq i} \mathbf{f}_{i j}^{\mathrm{pair}}+\mathrm{f}_{i}^{\mathrm{ext}}
$$

where

$$
\mathbf{f}_{i j}^{\mathrm{pair}}=\mathbf{f}_{i j}^{\mathrm{el}}+\mathbf{f}_{i j}^{\mathrm{rep}}
$$

and

$$
\mathbf{f}_{i}^{\mathrm{ext}}=\sum_{j} \mathbf{f}_{i j}^{\mathrm{el} \prime}+\mathbf{f}_{i, \text { wall }}^{\mathrm{rep}}
$$

Small amplitude oscillatory shear and stress relaxation ideally consider the rheological response when the spheres are displaced only infinitesimally from their equilibrium po- 
sitions. Hence we expand the force on each sphere in a Taylor series about the equilibrium configuration $\left\{\mathbf{r}_{k}^{0}\right\}$ (truncated after the linear term),

$$
\mathbf{f}_{i}^{\mathrm{tot}}\left(\left\{\mathbf{r}_{k}(t)\right\}\right)=\mathbf{f}_{i}^{\mathrm{fot}}\left(\left\{\mathbf{r}_{k}^{0}\right\}\right)+\left.\sum_{l} \Delta \mathbf{r}_{l}(t) \cdot \nabla_{l} \mathbf{f}_{i}^{\mathrm{ot}}\right|_{\left\{\mathbf{r}_{k}^{0}\right\}}
$$

where $\Delta \mathbf{r}_{l}(t)=\mathbf{r}_{l}(t)-\mathbf{r}_{l}^{0}$ is the small displacement of sphere $l$ from its equilibrium position. Since the spheres experience no net force at equilibrium, the first term on the right side of Eq. (43) is identically zero.

Substituting Eqs. (41)-(43) into Eq. (40) yields

$$
\begin{aligned}
\mathbf{f}_{i}^{\mathrm{tot}}\left(\left\{\mathbf{r}_{k}\right\}\right)= & \left.\sum_{j \neq i}\left[\Delta \mathbf{r}_{j}(t)-\Delta \mathbf{r}_{i}(t)\right] \cdot \nabla_{j} \mathbf{f}_{i j}^{\text {pair }}\right|_{\left\{\mathbf{r}_{k}^{0}\right\}}+\left.\Delta \mathbf{r}_{i}(t) \cdot \nabla_{i} \mathbf{f}_{i w}^{\text {rep }}\right|_{\left\{\mathbf{r}_{k}^{0}\right\}} \\
& +\sum_{j}^{*}\left[\left.\Delta \mathbf{r}_{j}(t) \cdot \nabla_{j} \mathbf{f}_{i j}^{\mathrm{el} \prime}\right|_{\left\{\mathbf{r}_{k}^{0}\right\}}+\left.\Delta \mathbf{r}_{i}(t) \cdot \nabla_{i} \mathbf{f}_{i j}^{\text {el }}\right|_{\left\{\mathbf{r}_{k}^{0}\right\}}\right]
\end{aligned}
$$

Here we have employed the identity $\nabla_{i}=-\nabla_{j}$ for pair forces. For the interactions between spheres and reflected images, the gradient operators obey the relationships $\mathbf{e}_{x} \cdot \nabla_{i}=-\mathbf{e}_{x} \cdot \nabla_{j}$, and $\mathbf{e}_{z} \cdot \nabla_{i}=\mathbf{e}_{z} \cdot \nabla_{j}$. The asterisk in the last summation indicates that the $i$ interaction is counted only once.

Substitution of this force expression into Eq. (8) provides a linearized equation of motion for sphere $i$ not near an electrode.

$$
\begin{aligned}
\frac{d \Delta \mathbf{r}_{i}(t)}{d t}= & \mathbf{u}^{\infty}\left(\mathbf{r}_{i}^{0}\right)+\frac{1}{\sigma_{i}}\left\{\left.\sum_{j \neq i}\left[\Delta \mathbf{r}_{j}(t)-\Delta \mathbf{r}_{i}(t)\right] \cdot \nabla_{j} f_{i j}^{\text {pair }}\right|_{\left\{\mathbf{r}_{k}^{0}\right\}}+\left.\Delta \mathbf{r}_{i}(t) \cdot \nabla_{i} \mathbf{f}_{i w}^{\text {rep }}\right|_{\left\{\mathbf{r}_{k}^{0}\right\}}\right. \\
& \left.+\sum_{j}^{*}\left[\left.\Delta \mathbf{r}_{j}(t) \cdot \nabla_{j} \mathbf{f}_{i j}^{\text {el' }}\right|_{\left\{\mathbf{r}_{k}^{0}\right\}}+\left.\Delta \mathbf{r}_{i}(t) \cdot \nabla_{i} \mathbf{f}_{i j}^{\text {el }}\right|_{\left\{\mathbf{r}_{k}^{0}\right]}\right]\right\}
\end{aligned}
$$

The $z$ component of this equation also describes the $z$ component of the motion of spheres stuck to the electrodes; the $x$ and $y$ motion of stuck spheres are constrained to follow the prescribed electrode displacements. Equation (45) represents a set of linear, first order, ordinary differential equations that can be solved exactly to provide the particle displacements, or positions, as a function of time. This analysis is valid for any configuration and any ambient flow, provided the deformation is sufficiently small and the initial metastable structure is known (e.g., obtained from the simulation method). We now obtain the solution of Eq. (45) for shear stress relaxation of the three-sphere cluster depicted in Fig. 15. To simplify this problem, we constrain spheres 1 and 2 to be fixed to the electrodes; their positions prior to the deformation are determined by simulation under no flow, and they translate only in the $x$ direction during the "step strain." These spheres remain fixed in their new positions during the relaxation process. Hence, their trajectories are prescribed, and only the solution for the trajectory of sphere 3 is required. We further simplify this problem by neglecting the interaction between sphere 3 and all images.

In this case, the total force on sphere 3 is due only to the pair interactions with spheres 1 and 2 , 


$$
\mathbf{f}_{3}^{\text {tot }}=\sum_{j=1}^{2}\left[\Delta \mathbf{r}_{j}(t)-\Delta \mathbf{r}_{3}(t)\right] \cdot \nabla_{j} \mathbf{f}_{3 j}^{\text {pair }},
$$

and the linearized equation of motion for sphere 3 becomes

$$
\frac{d \Delta \mathbf{r}_{3}(t)}{d t}=\mathbf{u}^{\infty}\left(\mathbf{r}_{3}^{0}\right)+\left.\frac{1}{\sigma_{3_{j}}=1} \sum^{2}\left[\Delta \mathbf{r}_{j}(t)-\Delta \mathbf{r}_{3}(t)\right] \cdot \nabla_{j} \mathbf{f}_{3 j}^{\mathrm{pair}}\right|_{\left\{\mathbf{r}_{k}^{0}\right\}},
$$

where the prescribed trajectories of spheres 1 and 2 are

$$
\Delta \mathbf{r}_{1}(t)=\left\{\begin{array}{cc}
\dot{\gamma} t L_{z} \mathbf{e}_{x} & 0<t<t_{0} \\
\dot{\gamma} t_{0} L_{z} \mathbf{e}_{x} & t>t_{0},
\end{array}\right.
$$

and

$$
\Delta \mathbf{r}_{2}(t)=0 .
$$

The initial configuration $\left\{\mathbf{r}_{k}^{0}\right\}$ is determined a priori, e.g., by simulation.

For the step strain portion of the relaxation experiment, the motion of sphere 3 is dominated by hydrodynamic drag, providing the following solution for $t<t_{0}$ :

$$
\Delta \mathbf{r}_{3}(t)=\dot{\gamma} t\left(z_{3}^{0}+\frac{L_{z}}{2}\right) \mathbf{e}_{x}, \quad t<t_{0}
$$

For the relaxation portion of the experiment, $t \geqslant t_{0}$, the ambient velocity vanishes, spheres 1 and 2 remain fixed, and Eq. (47) can be written as a set of two linear ordinary differential equations with constant coefficients, governing the motion of sphere 3:

$$
\frac{d \Delta x_{3}(t)}{d t}=a_{0}-a_{1} \Delta x_{3}(t)-a_{2} \Delta z_{3}(t)
$$

and

$$
\frac{d \Delta z_{3}(t)}{d t}=b_{0}-b_{1} \Delta x_{3}(t)-b_{2} \Delta z_{3}(t)
$$

with the "initial" conditions

$$
\Delta x_{3}\left(t=t_{0}\right)=\dot{\gamma} t_{0}\left(z_{3}^{0}+L_{z} / 2\right)
$$

and

$$
\Delta z_{3}\left(t=t_{0}\right)=0 .
$$

The constant coefficients are expressed as functions of the equilibrium positions and the prescribed electrode displacements,

$$
\begin{aligned}
a_{0}= & \frac{1}{\sigma_{3}}\left[\Delta x_{1}\left(t_{0}\right) \frac{\partial}{\partial x_{1}} f_{x, 31}^{\text {pair }}+\Delta x_{2}\left(t_{0}\right) \frac{\partial}{\partial x_{2}} f_{x, 32}^{\text {pair }}+\Delta z_{1}\left(t_{0}\right) \frac{\partial}{\partial z_{1}} f_{x, 31}^{\text {pair }}\right. \\
& \left.+\Delta z_{2}\left(t_{0}\right) \frac{\partial}{\partial z_{2}} f_{x, 32}^{\text {pair }}\right]
\end{aligned}
$$




$$
\begin{gathered}
a_{1}=\frac{1}{\sigma_{3}}\left(\frac{\partial}{\partial x_{1}} f_{x, 31}^{\text {pair }}+\frac{\partial}{\partial x_{2}} f_{x, 32}^{\text {pair }}\right) \\
a_{2}=\frac{1}{\sigma_{3}}\left(\frac{\partial}{\partial z_{1}} f_{x, 31}^{\text {pair }}+\frac{\partial}{\partial z_{2}} f_{x, 32}^{\text {pair }}\right), \\
b_{0}=\frac{1}{\sigma_{3}}\left[\Delta x_{1}\left(t_{0}\right) \frac{\partial}{\partial x_{1}} f_{x, 31}^{\text {pair }}+\Delta x_{2}\left(t_{0}\right) \frac{\partial}{\partial x_{2}} f_{x, 32}^{\text {pair }}+\Delta z_{1}\left(t_{0}\right) \frac{\partial}{\partial z_{1}} f_{x, 31}^{\text {pair }}\right. \\
\left.+\Delta z_{2}\left(t_{0}\right) \frac{\partial}{\partial z_{2}} f_{x, 32}^{\text {pair }}\right], \\
b_{1}=\frac{1}{\sigma_{3}}\left(\frac{\partial}{\partial x_{1}} f_{z, 31}^{\text {pair }}+\frac{\partial}{\partial x_{2}} f_{z, 32}^{\text {pair }}\right)
\end{gathered}
$$

and

$$
b_{2}=\frac{1}{\sigma_{3}}\left(\frac{\partial}{\partial z_{1}} f_{z, 31}^{\mathrm{pair}}+\frac{\partial}{\partial z_{2}} f_{z, 32}^{\mathrm{pair}}\right),
$$

where the force derivatives are evaluated at the equilibrium configuration. Symmetry in this particular configuration results in $a_{2}=b_{1}=0$. The solution for the trajectory of sphere 3 for $t \geqslant t_{0}$ is

$$
\Delta x_{3}(t)=\frac{a_{0}}{a_{1}}+\left[\dot{\gamma} t_{0}\left(z_{3}^{0}+\frac{L_{z}}{2}\right)-\frac{a_{0}}{a_{1}}\right] e^{-a_{1}\left(t-t_{0}\right)},
$$

and

$$
\Delta z_{3}(t)=\frac{b_{0}}{b_{2}}\left(1-e^{-b_{2}\left(t-t_{0}\right)}\right) .
$$

The relaxation modulus is obtained by substituting the trajectories into the linearized force expression $[\mathrm{Eq}$. (44) with the simplifications outlined at the beginning of this appendix], and then calculating the shear stress [Eq. (16)] and modulus [Eq. (17)]. To leading order, the relaxation modulus for this structure is a linear combination of the two exponential functions $e^{-a_{1} t}$ and $e^{-b_{2} t}$. Although two relaxation times arise in this analysis, relaxation is dominated by the motion of sphere 3 in the $z$ direction, and the only significant peak in the relaxation spectrum arises at $\tau=1 / b_{2}$.

In the present study, relaxation spectra are approximated from the relaxation modulus through Eq. (26). However, this perturbation scheme will in general produce relaxation moduli expressed as linear combinations of exponential functions, from which the corresponding spectra can be determined directly in terms of delta functions.

\section{References}

Bailey, R., D. G. Gillies, D. M. Heyes, and L. II. Sutcliffe, "Experimental and simulation studies of electrorheology," Mol. Sim. 4, 137-151 (1989).

Bonnecaze, R. T., and J. F. Brady, "Dynamic simulation of a suspension of dielectric particles forming an electrorheological fluid," Proceedings of the Second International Conference on ER Fluids, edited by J. D. Carlson, A. F. Sprecher, and H. Conrad (Technomic, Lancaster, PA, 1990), pp. 27-40. 
Bonnecaze, R. T. and J. F Brady, "Dynamic simulation of an electrorheological fluid," J. Chem. Phys. 96, 2183-2202 (1992a).

Bonnecaze, R. T. and J. F. Brady, "Yield stresses in electrorheological fluids," J. Rheol. 36, 73-115 (1992b)

Conrad, H., personal communication (1994).

Elster, C. and J. Honerkamp, "Modified maximum entropy method and its application to creep data," Macromolecules 24, 310-314 (1991).

Ferry, J. D., Viscoelastic Properties of Polymers, 3rd ed. (Wiley, New York, 1980).

Honerkamp, J, and J. Weese, "Determination of the relaxation spectrum by a regularization method," Macromolecules 22, 4372-4377 (1989).

Klingenberg, D. J., "Simulation of the dynamic oscillatory response of electrorheological suspensions: Demonstration of a relaxation mechanism," J. Rheol. 37, 199-214 (1993).

Klingenberg, D. J., F. van Swol, and C. F. Zukoski, "Dynamic simulation of electrorheological suspensions," J. Chem. Phys. 91, 7888-7895 (1989).

Klingenberg, D. J., F. van Swol, and C. F. Zukoski, "The small shear rate response of electrorheological suspensions: I. Simulation in the point-dipole limit," J. Chem. Phys. 94, 6160-6169 (1991a).

Klingenberg, D. J., F. van Swol, and C. F. Zukoski, "The small shear rate response of electrorheological suspensions: I. Extension beyond the point-dipole limit, J. Chem. Phys. 94, 6170-6178 (1991b).

Klingenberg, D. J. and C. F. Zukoski, "Studies on the steady-shear behavior of electrorheological suspensions," Langmuir 6, 15-24 (1990).

Marshall, L., J. Goodwin, and C. F. Zukoski, "Effects of electric fields on the rheology of nonaqueous concentrated suspensions," J. Chem. Soc. Faraday Trans. I 85, 2785-2795 (1989).

McLeish, T. C. B., T. Jordan, and M. T. Shaw, "Viscoelastic response of electrorheological fluids. I. Frequency dependence," J. Rheol. 35, 427-448 (1991).

Melrose, J. R., "Brownian dynamics simulation of dipole suspensions under shear: the phase diagram," Mol. Phys. 76, 635-660 (1992).

Otsubo, Y., M. Sekine, and S. Katayama, "Electrorheological properties of silica suspensions," J. Rheol. 36, 479-496 (1992).

Parthasarallyy, M., K. H. Aln, B. Belongia, and D. J. Klingenberg, "The role of suspension structure in the dynamic response of electrorheological suspensions," presented at the Fourth International Conference on Electrorheological Fluids, Feldkirch, Austria, July 20-23, 1993.

Russel, W. B., D. A. Saville, and W. R. Schowalter, Colloidal Dispersions (Cambridge University, Cambridge, 1989).

See, H. and M. Doi, "Shear resistance of electrorheological fluids under time-varying electric fields," J. Rheol 36, 1143-1163 (1992).

Sprecher, A. F., Y. Chen, and H. Conrad, "Measurement of forces between particles in a model ER fluid," Proceedings of the Second International Conference on ER Fluids, edited by J. D. Carlson, A. F. Sprecher and H. Conrad (Technomic, Lancaster, PA, 1990), pp. 82-89.

Tschoegl, N. W., The Phenomenological Theory of Linear Viscoelastic Behavior (Springer, London, 1989).

Whittle, M., "Computer simulation of an electrorheological fluid," J. Non-Newtonian Fluid Mech. 37, 233-263 (1990) 